\title{
ON SEMIGROUPS OF ENDOMORPHISMS OF GENERALIZED BOOLEAN RINGS
}

\author{
DOUGLAS B. SMITH, JR. and JIANG LUH
}

(Received 12 February 1973)

Communicated by G. B. Preston

\section{Introduction}

Magill in [4] first proved that two Boolean rings are isomorphic if and only if their respective endomorphism semigroups are isomorphic. His proof, however, relied on topological techniques. More recently Maxson has published a proof of the above using purely algebraic techniques [5]. In this paper, structure theorems are given which allow us to extend the above result to the $p^{k}$-rings of Foster [1]. As a special case, the result is shown to apply also to $p$-rings. An example is given to show that a further extension to $J$-rings is impossible.

Throughout this paper a $p$-ring will be a ring $R$ with unity $1_{R}$ of characteristic $p$, where $p$ is prime, and having the property that $x^{p}=x$ for all $x \in R$. We will consider two types of $p^{k}$-rings, the type always being identified by its author's name. Let $p$ be a prime integer and $k$ a positive integer. Then a $p^{k}$-ring (McCoy) $R$ is a ring with unity $1_{R}$ of characteristic $p$ such that $x^{p k}=x$ for all $x \in R$. These were first introduced in [6]. The following more restrictive definition was introduced by Foster in [1]. Again let $p$ be a prime integer and $k$ a positive integer. A ring $R$ is a $p^{k}$-ring (Foster) if the following hold:

(i) $1_{R} \in R$

(ii) $x^{p k}=x$ for all $x \in R$

(iii) $R$ has at least one subring $F$ which is isomorphic to the Galois field of $p^{k}$ elements, $G F\left(p^{k}\right)$, and

(iv) $1_{R} \in F$.

Any subring $F$ of a $p^{k}$-ring (Foster) satisfying (iii) and (iv) is called a normal subfield of $R$.

Note that since $1_{R} \in F$ and $F$ is of characteristic $p, R$ is of characteristic $p$, and hence a $p^{k}$-ring (Foster) is a $p^{k}$-ring (McCoy). The reverse is not true, as illustrated by the ring $G F(2) \oplus G F\left(2^{2}\right)$, which is a $p^{k}$-ring (McCoy) but not a $p^{k}$-ring (Foster). Both types of $p^{k}$-rings are $p$-rings when $k=1$. We observe also 
that if $R$ is a $p^{k}$-ring (Foster) and $F$ is a normal subfield of $R$, then $R$ is an algebra over $F$.

A $J$-ring is any ring $R$ for which there exists an integer $n>1$ such that $x^{n}=x$ for all $x \in R$.

Each type of ring we have defined is commutative (cf. [3] page 217), so the set of idempotents $R^{\prime}$ of such a ring $R$ is easily seen to be a semigroup under multiplication. The set of ring endomorphisms of $R$, End $R$, is a semigroup under composition of functions. Thinking of a $p^{k}$-ring (Foster) as an algebra over some normal subfield $F$, the set of algebra endomorphisms of $R$ over $F$, denoted by $\operatorname{End}_{F} R$, is also a semigroup under composition of functions.

The mapping $e \rightarrow \phi_{e}$, where $\phi_{e}(r)=e r$ for all $r \in R$, is easily seen to embed $R^{\prime}$ in End $R$ for each of the rings discussed above. If $R$ is a $p^{k}$-ring (Foster) and $F$ a normal subfield of $R$, then the same mapping embeds $R^{\prime}$ in $\operatorname{End}_{F} R$.

\section{2. $p^{k}$-rings}

We now present some structure theorems for the $p^{k}$-rings of McCoy and Foster. McCoy in [7] has shown that if $R$ is a $p$-ring, then $R$ is isomorphic to a subdirect sum of fields $G F(p)$, and that if $R$ is a $p^{k}$-ring (McCoy), then $R$ is isomorphic to a subdirect sum of fields of the form $G F\left(p^{k_{i}}\right)$. If $R$ is a $p^{k}$-ring (McCoy) and $S$ a homomorphic image of $R$, then $S$ is a $p^{k}$-ring (McCoy). Further, if $S$ is subdirectly irreductible, then $S$ is isomorphic to $G F\left(p^{t}\right)$, where $t \mid k$.

THEOREM 2.1. Any nonzero homomorphic image of a $p^{k}$-ring (Foster) is a $p^{k}$-ring (Foster).

Proof. Suppose $\theta: R \rightarrow S$ is an epimorphism, where $R$ is a $p^{k}$-ring (Foster). If $x \in S$ then obviously $x^{p^{k}}=x$. If $F$ is a normal subfield of $R$, then necessarily $\theta(F) \simeq F \simeq G F\left(p^{k}\right) .1_{R} \in F$ so $1_{S}=\theta\left(1_{R}\right) \in \theta(F) \subseteq S$ and $S$ is a $p^{k}$-ring (Foster).

The following theorem forms the basis for the main result of this paper.

THEOREM 2.2. If $R$ is a $p^{k}$-ring (Foster) and $F$ a normal subfield of $R$, then each element $r \in R$ can be uniquely expressed in the form

$$
r=\sum_{i} \alpha_{i} x_{i}
$$

where the $\alpha_{i}$ are the nonzero elements of $F$ and the $x_{i}$ are idempotent elements of $R$ such that $x_{m} x_{n}=0$ if $m \neq n$ and $\sum_{i} x_{i}=1_{R}$.

The proof of this theorem, in a somewhat more general setting, may be found in $[2]$.

As a result of this structure theorem we have the following theorem.

THEOREM 2.3. If $R$ is a subdirect sum of finitely many $p_{i}^{k^{i}}{ }^{-r i n g s}$ (Foster) then $R$ is isomorphic to a direct sum of some of these same rings.

Proof. Let $R$ be a subdirect sum of rings $M_{i}(i=1,2, \cdots, n)$, where $M_{i}$ is a 
$p_{i}^{k_{i}}$-ring (Foster) containing a normal subfield $F_{i} \simeq G F\left(p_{i}^{k_{i}}\right)$. We prove the theorem by induction on $n$. Clearly the theorem is true for $n=1$. Suppose now that the theorem holds for all rings that are subdirect sums of $k-1 \geqq 1$ rings, and suppose that $R$ is a subdirect sum of $p_{i}^{k_{i}}$-rings (Foster) $M_{i}(i=1,2, \cdots, k)$. Let $\mu: R \rightarrow \sum_{i=1}^{k} \oplus M_{i}$ be a monomorphism and $\pi_{j}: \sum_{i=1}^{k} \oplus M_{i} \rightarrow M_{j}$ be the projection epimorphism such that $\pi_{j} \mu$ is an epimorphism for each $j=1,2, \cdots, k$. Define $T_{i}=\left\{\mu(x) \mid x \in R\right.$ and $\pi_{j} \mu(x)=0$ for all $\left.j \neq i\right\}$ for each $i=1,2, \cdots, k$. We consider two cases.

CASE 1. For each $i, T_{i} \neq\{0\}$. Then for each $i$ there exists a nonzero $a_{i} \in M_{i}$ such that $\left(0, \cdots, 0, a_{i}, 0, \cdots, 0\right) \in \mu(R)$, where $a_{i}$ is the $i$ th component. Now $M_{i}$ is a $p_{i}^{k_{i}}$-ring (Foster), so by 2.2, $a_{i}=\Sigma_{m} \alpha_{m} x_{m}$, where the $\alpha_{m}$ are the nonzero elements of $F_{i}$ and the $x_{m}$ the appropriate idempotent elements in $M_{i}$. Since for each $m, \alpha_{m}^{-1} x_{m} \in M_{i}$, there exists an $r \in R$ such that $\pi_{i} \mu(r)=\alpha_{m}^{-1} x_{m}$, and consequently there is an element in $\sum_{i=1}^{k} \oplus M_{i}$, say $\left(b_{1}^{(m)}, b_{2}^{(m)}, \cdots, b_{i}^{(m)}, \cdots, b_{k}^{(m)}\right.$ ) $=\mu(r)$, where $b_{i}^{(m)}=\alpha_{m}^{-1} x_{m}$. Thus $\left(0, \cdots, 0, x_{m}, 0, \cdots, 0\right)=\left(0, \cdots, 0, a_{i}, 0, \cdots, 0\right)$ $\left(b_{1}^{(m)}, b_{2}^{(m)}, \cdots, b_{i}^{(m)}, \cdots b_{k}^{(m)}\right) \in \mu(R)$, where $x_{m}$ is the $i$ th component. This is true for each $m$, so the sum of all such elements is in $\mu(R)$. But $\Sigma_{m} x_{m}=1_{R}$, so $\left(0, \cdots, 0,1_{R}, 0, \cdots, 0\right)$, where $1_{R}$ is the $i$ th component is in $\mu(R)$. Since $i$ was arbitrary we have $\mu(R)=\sum_{i=1}^{k} \oplus M_{i}$, and $R$ is isomorphic to a direct sum of the $M_{i}$.

CASE 2. $T_{i}=\{0\}$ for some $i$. Without loss of generality, assume $T_{k}=\{0\}$. We define a map $\phi$ of $\mu(R)$ into the direct sum $\sum_{i=1}^{k} \oplus M_{i}$ by $\phi\left(x_{1}, x_{2}, \cdots, x_{k-1}, x_{k}\right)$ $=\left(x_{1}, x_{2}, \cdots, x_{k-1}\right)$. Since $T_{k}=\{0\}, \phi$ is a monomorphism. Hence $\phi \mu$ is a monomorphism of $R$ into $\sum_{i=1}^{k-1} \oplus M_{i}$ and $\pi_{j} \phi \mu$ is an epimorphism for $j=1,2, \cdots, k-1$. $R$ is thus a subdirect sum of $M_{1}, \cdots, M_{k-1}$, so by the inductive assumption, $R$ is a direct sum of some of the $M_{1}, \cdots, M_{k-1}$.

CoRollary 2.4. (Foster) If $R$ is a finite $p^{k}$-ring (Foster), then $R$ is isomorphic to a direct sum of finitely many copies of $G F\left(p^{k}\right)$.

Proof. This is an immediate consequence of Theorem 2.3 and that of the note which precedes Theorem 2.1.

\section{Endomorphisms of $p^{k}$-rings}

Throughout this section let $p$ be a fixed prime integer, $k$ a fixed positive integer, $R$ and $S p^{k}$-rings (Foster) with normal subfields $F$ and $G$ respectively, and $R^{\prime}$ and $S^{\prime}$ the semigroups of idempotents of $R$ and $S$, respectively. We will show that if $\operatorname{End}_{F} R \simeq \operatorname{End}_{G} S$ as semigroups, then $R^{\prime} \simeq S^{\prime}$ as semigroups.

We will identify $R^{\prime}$ and $S^{\prime}$ with their isomorphic images in $\operatorname{End}_{F} R$ and $\operatorname{End}_{G} S$, respectively. The elements of $R^{\prime}$ will be denoted by $\phi_{r}$, where $r=r^{2} \in R$, and those of $S^{\prime}$ by $\psi_{s}$, where $s=s^{2} \in S$. Specifically the zero and unit el.m nts of $R^{\prime}$ will be $\phi_{0}$ and $\phi_{1}$, while those of $S^{\prime}$ will be $\psi_{0}$ and $\psi_{1}$. 
In some of the proofs that follow, we will refer, for example, to $\phi_{e}+\phi_{r}$, where $e=e^{2}, r=r^{2} \in R$, although addition is not defined in End $R$. We can legitimately do this if we consider $\phi_{e}$ and $\phi_{r}$ as elements of the $\operatorname{ring} \operatorname{End}(R,+)$, where we are considering all endomorphisms of the abelian group $(R,+)$.

Let $\pi: \operatorname{End}_{F} R \rightarrow \operatorname{End}_{G} S$ be a semigroup isomorphism.

Lemma 3.1. $\pi\left(\phi_{0}\right)=\psi_{0}$ and $\pi\left(\phi_{1}\right)=\psi_{1}$.

LEMMA 3.2. If $\psi_{s} \in S^{\prime}, \phi=\pi^{-1}\left(\psi_{s}\right)$, and $\phi_{e} \in R^{\prime}$, then $\phi \phi_{e}=\phi_{e} \phi$.

Proof. Note that $\phi_{1}-\phi_{e}=\phi_{1-e} \in R^{\prime} \subseteq \operatorname{End}_{F} R$, so $\phi_{e} \phi\left(\phi_{1}-\phi_{e}\right) \in \operatorname{End}_{F} R$ We show now that $\phi_{e} \phi\left(\phi_{1}-\phi_{e}\right)=\phi_{0}$.

$$
\begin{aligned}
{\left[\pi\left(\phi_{e} \phi\left(\phi_{1}-\phi_{e}\right)\right)\right]\left(1_{s}\right) } & =\left[\pi\left(\phi_{e}\right) \psi_{s} \pi\left(\phi_{1}-\phi_{e}\right)\right]\left(1_{s}\right)=\pi\left(\phi_{e}\right)\left\{s \cdot\left[\pi\left(\phi_{1}-\phi_{e}\right)\right]\left(1_{s}\right)\right\} \\
& =\left[\pi\left(\phi_{e}\right)(s)\right]\left[\pi\left(\phi_{e}-\phi_{e}\right)\left(1_{s}\right)\right]=\left[\pi\left(\phi_{e}\right)(s)\right]\left[\psi_{0}\left(1_{s}\right)\right]=0 .
\end{aligned}
$$

Thus $\pi\left(\phi_{e} \phi\left(\phi_{1}-\phi_{e}\right)\right)=\psi_{0}$ and hence $\phi_{e} \phi\left(\phi_{1}-\phi_{e}\right)=\phi_{0}$, so $\phi_{e} \phi=\phi_{e} \phi \phi_{e}$. Similarly $\phi \phi_{e}=\phi_{e} \phi \phi_{e}$. Thus, $\phi \phi_{e}=\phi_{e} \phi$.

LEMMA 3.3. If $\psi_{s} \in S^{\prime}$ and $\phi=\pi^{-1}\left(\psi_{s}\right)$ then $\phi\left(e e^{\prime}\right)=e \phi\left(e^{\prime}\right)$ for all $e=e^{2}$, $e^{\prime}=\left(e^{\prime}\right)^{2} \in R$.

Proof. $\phi\left(e e^{\prime}\right)=\phi \phi_{e}\left(e^{\prime}\right)=\phi_{e} \phi\left(e^{\prime}\right)=e \phi\left(e^{\prime}\right)$ by 3.2 since $\phi_{e} \in R^{\prime}$.

LEMMA 3.4. If $\psi_{s} \in S^{\prime}$ and $\phi=\pi^{-1}\left(\psi_{s}\right)$, then $\phi\left(r r^{\prime}\right)=\phi(r) r^{\prime}$, for all $r$, $r^{\prime} \in R$.

ProOF. By 2.2 we may uniquely write $r$ and $r^{\prime}$ as $r=\Sigma_{i} \alpha_{i} x_{i}, r^{\prime}=\Sigma_{k} \beta_{j} x^{\prime}{ }_{j}$, where $\alpha_{i}, \beta_{j} \in F$ and $x_{i}=\left(x_{i}\right)^{2}, x_{j}^{\prime}=\left(x_{j}^{\prime}\right)^{2} \in R$ are such that $x_{m} x_{n}=x_{m}^{\prime} x_{n}^{\prime}=0$ if $m \neq n$ and $\sum_{i} x_{i}=\Sigma_{j} x_{j}=1_{R}$.

Thus

$$
\begin{aligned}
\phi\left(r r^{\prime}\right) & =\phi\left(\sum_{i} \alpha_{i} x_{i} \sum_{j} \beta_{j} x_{j}^{\prime}\right)=\phi\left(\sum_{i, j} \alpha_{i} \beta_{j} x_{i} x_{j}^{\prime}\right) \\
& =\sum_{i, j} \phi\left(\alpha_{i} \beta_{j}\right) \phi\left(x_{i} x_{j}^{\prime}\right) \text { since } \phi \in \operatorname{End}_{F} R \\
& =\sum_{i, j} \phi\left(\alpha_{i}\right) \beta_{j} \phi\left(x_{i}\right) x_{j}^{\prime} \text { since } \phi \in \operatorname{End}_{F} R \text { and by } 3.3 \\
& =\sum_{i} \phi\left(\alpha_{i} x_{i}\right) \sum_{j} \beta_{j} x_{j}^{\prime}=\phi(r) r^{\prime} .
\end{aligned}
$$

LeMMA 3.5. If $\psi_{\mathrm{s}} \in S^{\prime}$ and $\phi=\pi^{-1}\left(\psi_{\mathrm{s}}\right)$, then $\phi \in R^{\prime}$.

PRoof. If $r \in R$ then $\phi(r)=\phi\left(1_{R} \cdot r\right)=\phi\left(1_{R}\right) \cdot r$ by 3.4. Thus if $e=\phi\left(1_{R}\right)$ then $e=e^{2}$ and $\phi=\phi_{\epsilon} \in R^{\prime}$.

THEOREM 3.6. If $\operatorname{End}_{F} R \simeq \operatorname{End}_{G} S$ then $R^{\prime} \simeq S^{\prime}$. 
Proof. By $3.5, \pi^{-1}\left(S^{\prime}\right) \subseteq R^{\prime}$ so $S^{\prime} \subseteq \pi\left(R^{\prime}\right)$. By a similar argument we can show that $\pi\left(R^{\prime}\right) \subseteq S^{\prime}$, giving $S^{\prime} \subseteq \pi\left(R^{\prime}\right) \subseteq S^{\prime}$, so $\pi\left(R^{\prime}\right)=S^{\prime}$. Since $\pi$ preserves multiplication and is one-one, the theorem is proved.

\section{The main theorem}

Let $p$ be a fixed prime integer, $k$ a fixed positive integer, and $R$ and $S p^{k}$-rings (Foster) with normal subfields $F$ and $G$, respectively. Let $R^{\prime}$ and $S^{\prime}$ be the semigroups of idempotents of $R$ and $S$, respectively, and let $\pi: R^{\prime} \rightarrow S^{\prime}$ be a semigroup isomorphism. Since $F \simeq G F\left(p^{k}\right) \simeq G$, let $\sigma: F \rightarrow G$ be a field isomorphism. We will use the next two lemmas freely, without specific reference to them.

LEMMA 4.1. $\pi(0)=0$ and $\pi\left(1_{R}\right)=1_{S}$.

Proof. The proof is basically the same as that of 3.1.

Lemma 4.2. If $x \in R^{\prime}$ then $\pi\left(1_{R}-x\right)=1_{S}-\pi(x)$.

Proof. Trivially $1_{R}-x \in R^{\prime}$ if $x \in R^{\prime}$. Suppose $\pi\left(1_{R}-x\right)=1_{S}-s$ for some $s \in S$. Then since $\pi\left(1_{R}-x\right) \in S^{\prime}, s=1_{S}-\pi\left(1_{R}-x\right) \in S^{\prime}$. Hence $s=\pi(y)$ for some $y \in R^{\prime}$, i.e.,

$$
\pi\left(1_{R}-x\right)=\pi\left(1_{R}\right)-\pi(y),
$$

so that by multiplying by $\pi(x)$ we have $0=\pi(x)-\pi(x y)$. Since $\pi$ is one-one, $x=x y$. Multiplying (1) by $\pi(y)$ gives $y=x y$, so $x=y$.

Lemma 4.3. Suppose that $\alpha \in F, x \in R^{\prime}$, and $\alpha x \in R^{\prime}$. Then $\pi(\alpha x)=\sigma(\alpha) \pi(x)$.

Proof. If $x=0$ the conclusion is obvious. Suppose $x \neq 0$. Then since $\alpha x, x \in R^{\prime}, \alpha x=(\alpha x)^{2}=\alpha^{2} x$, so

$$
\left(\alpha^{2}-\alpha\right) x=0 .
$$

Now since $\alpha^{2}-\alpha \in F, \alpha^{2}-\alpha=0$, else we could multiply (2) by $\left(\alpha^{2}-\alpha\right)^{-1}$ and obtain $x=0$. But $\alpha(\alpha-1)=0$ implies $\alpha=0$ or $\alpha=1$ because $F$ is a field. Since $\sigma$ is a field isomorphism, $\sigma(0)=0$ and $\sigma(1)=1$, the conclusion following immediately.

LeMMA 4.4. Let $x_{1}, x_{2}, \cdots, x_{n} \in R^{\prime}$ and $\alpha_{1}, \alpha_{2}, \cdots, \alpha_{n} \in F$. If

$$
\sum_{i=1}^{n} \alpha_{i}\left(x_{1} x_{i}\right) \in R^{\prime} \text { then } \pi\left[\sum_{i=1}^{n} \alpha_{i}\left(x_{1} x_{i}\right)\right]=\sum_{i=1}^{n} \sigma\left(\alpha_{i}\right) \pi\left(x_{1} x_{i}\right)
$$

Proof. We proceed by induction. By 4.3 the conclusion holds for $n=1$. Suppose the lemma is true for $n=k$. Then 


$$
\begin{aligned}
\pi\left[\sum_{i=1}^{k+1} \alpha_{i}\left(x_{1} x_{i}\right)\right]= & \pi\left[\sum_{i=1}^{k+1} \alpha_{i}\left(x_{1} x_{i}\right)\right]\left[\pi\left(x_{1} x_{k+1}\right)+\pi\left(1_{R}\right)-\pi\left(x_{1} x_{k+1}\right)\right] \\
= & \pi\left[\left(\sum_{i=1}^{k+1} \alpha_{i} x_{1} x_{i}\right)\left(x_{1} x_{k+1}\right)\right]+\pi\left[\left(\sum_{i=1}^{k+1} \alpha_{i} x_{1} x_{i}\right)\left(1_{R}-x_{1} x_{k+1}\right)\right] \\
= & \pi\left[\sum_{i=1}^{k+1} \alpha_{i} x_{1} x_{i} x_{k+1}\right]+\pi\left[\sum_{i=1}^{k+1} \alpha_{i} x_{1} x_{i}-\sum_{i=1}^{k+1} \alpha_{i} x_{1} x_{i} x_{k+1}\right] \\
= & \pi\left[\left(\alpha_{1}+\alpha_{k+1}\right) x_{1} x_{k+1}+\sum_{i=2}^{k} \alpha_{i} x_{1} x_{i} x_{k+1}\right] \\
& +\pi\left[\sum_{i=1}^{k} \alpha_{i} x_{1} x_{i}\left(1_{R}-x_{k+1}\right)\right]
\end{aligned}
$$

Since each of the quantities enclosed by brackets is in $R^{\prime}$ and in a form which allows us to use our inductive assumption, we do to obtain

$$
\begin{aligned}
\sigma\left(\alpha_{1}\right. & \left.+\alpha_{k+1}\right) \pi\left(x_{1} x_{k+1}\right)+\sum_{i=2}^{k} \sigma\left(\alpha_{i}\right) \pi\left(x_{1} x_{i} x_{k+1}\right)+\sum_{i=1}^{k} \sigma\left(\alpha_{i}\right) \pi\left(x_{1} x_{i}\right)\left(1_{s}-\pi\left(x_{k+1}\right)\right) \\
& =\sum_{i=1}^{k+1} \sigma\left(\alpha_{i}\right) \pi\left(x_{1} x_{i}\right)
\end{aligned}
$$

after cancellation, using 4.2 and the additivity of $\sigma$.

LEMma 4.5. If $x_{1}, x_{2}, \cdots, x_{n} \in R^{\prime}, \alpha_{1}, \alpha_{2}, \cdots, \alpha_{n} \in F$, and $\sum_{i=1} \alpha_{i} x_{i} \in R^{\prime}$, then $\pi\left[\sum_{i=1}^{n} \alpha_{i} x_{i}\right]=\sum_{i=1}^{n} \sigma\left(\alpha_{i}\right) \pi\left(x_{i}\right)$.

Proof. Again we proceed by induction. The lemma is true for $n=1$ by 4.3 . We now suppose the lemma to be true for $n=k$. Then following a technique similar to the proof of 4.4 we have

$$
\begin{aligned}
\pi\left[\sum_{i=1}^{k+1} \alpha_{i} x_{i}\right]= & {\left[\pi\left(\sum_{i=1}^{k+1} \alpha_{i} x_{i}\right)\right]\left[\pi\left(x_{1}\right)+\pi\left(1_{R}\right)-\pi\left(x_{1}\right)\right]=\pi\left[\sum_{i=1}^{k+1} \alpha_{i} x_{i} x_{1}\right] } \\
& +\pi\left[\sum_{i=1}^{k+1} \alpha_{i} x_{i}\left(1_{R}-x_{1}\right)\right] \\
= & \sum_{i=1}^{k+1} \sigma\left(\alpha_{i}\right) \pi\left(x_{i} x_{1}\right)+\pi\left[\sum_{i=2}^{k+1} \alpha_{i} x_{i}\left(1_{R}-x_{1}\right)\right] \\
= & \sum_{i=1}^{k+1} \sigma\left(\alpha_{i}\right) \pi\left(x_{i} x_{1}\right)+\sum_{i=2}^{k+1} \sigma\left(\alpha_{i}\right) \pi\left[x_{i}\left(1_{R}-x_{1}\right)\right] \\
\text { by the inductive hypothesis } & \sum_{i=1}^{k+1} \sigma\left(\alpha_{i}\right) \pi\left(x_{i}\right) \text { after cancellations. }
\end{aligned}
$$


THEOREM 4.6. If $R^{\prime}$ and $S^{\prime}$ are isomorphic as semigroups, then $R$ and $S$ are isomorphic as rings.

Proof. We define a function $\pi^{*}: R \rightarrow S$ as follows: If $r \in R$ has as its unique representation $r=\Sigma_{i} \alpha_{i} x_{i}$ guaranteed by 2.2 , let

$$
\pi^{*}(r)=\sum_{i} \sigma\left(\alpha_{i}\right) \pi\left(x_{i}\right)
$$

Note that the image of $r$ is indeed a legitimate representation of an element of $S$ - in particular $\Sigma_{i} \pi\left(x_{i}\right)=1_{s}$ by 4.1 and 4.5 . By the uniqueness of the representation of $r, \pi^{*}$ is a one-one function and obviously onto.

To show that $\pi^{*}$ is additive, let $r=\sum_{i} \alpha_{i} x_{i}, r^{\prime}=\sum_{i} \alpha_{i} x_{i}^{\prime}$, and $r+r^{\prime}$ $=\sum_{i} \alpha_{i} x_{i}^{\prime \prime}$ be the unique representations. Then

Multiplying by $\alpha_{k}^{-1} x_{k}^{\prime \prime}$ we have

$$
\sum_{i} \alpha_{i} x_{i}^{\prime \prime}=\sum_{i} \alpha_{i} x_{i}+\sum_{i} \alpha_{i} x_{i}^{\prime}
$$

$$
x_{k}^{\prime \prime}=\sum_{i} \alpha_{k}^{-1} \alpha_{i} x_{i} x_{k}^{\prime \prime}+\sum_{i} \alpha_{k}^{-1} \alpha_{i} x_{i}^{\prime} x_{k}^{\prime \prime} \in R^{\prime} .
$$

Thus by 4.5

$$
\pi\left(x_{k}^{\prime \prime}\right)=\sum_{i} \sigma\left(\alpha_{k}^{-1}\right) \sigma\left(\alpha_{i}\right) \pi\left(x_{i}\right) \pi\left(x_{k}^{\prime \prime}\right)+\sum_{i} \sigma\left(\alpha_{k}^{-1}\right) \sigma\left(\alpha_{i}\right) \pi\left(x_{i}^{\prime}\right) \pi\left(x_{k}^{\prime \prime}\right)
$$

and since $\sigma$ is a field isomorphism,

$$
\sigma\left(\alpha_{k}\right) \pi\left(x_{k}^{\prime \prime}\right)=\pi\left(x_{k}^{\prime \prime}\right)\left[\sum_{i} \sigma\left(\alpha_{i}\right) \pi\left(x_{i}\right)+\sum_{i} \sigma\left(\alpha_{i}\right) \pi\left(x_{i}^{\prime}\right)\right] .
$$

Summing over all $k$ and using the fact that $\Sigma_{k} \pi\left(x_{k}^{\prime \prime}\right)=1_{s}$, we have

$$
\begin{aligned}
\pi^{*}\left(r+r^{\prime}\right)=\sum_{k} \sigma\left(\alpha_{k}\right) \pi\left(x_{k}^{\prime \prime}\right) & =\sum_{i} \sigma\left(\alpha_{i}\right) \pi\left(x_{i}\right)+\sum_{i} \sigma\left(\alpha_{i}\right) \pi\left(x_{i}^{\prime}\right) \\
& =\pi^{*}(r)+\pi^{*}\left(r^{\prime}\right) .
\end{aligned}
$$

A similar technique shows $\pi^{*}$ to be multiplicative, and thus an isomorphism.

Corollary 4.7. If $E n d_{F} R \simeq E_{\text {End }} S$ then $R \simeq S$.

Proor. This follows immediately from 3.6 and 4.6.

Note that each $p$-ring $R$ is a $p^{k}$-ring in the sense of Foster, the normal subfield $F$ being isomorphic to $G F(p)$. Further $R$ is an algebra over $F$ and $\operatorname{End}_{F} R=$ End $R$. With this in mind we have

COROLlaRY 4.8. Let $p$ be a fixed prime integer. If $R$ and $S$ are p-rings such that End $R \simeq$ End $S$, then $R \simeq S$.

\section{Remarks}

It is not known whether the Corollary 4.8 can be extended to the $p^{k}$-rings of Foster, wherein the entire semigroups of ring endomorphisms are used, to the 
$p^{k}$-rings of McCoy, or to direct sums of $p^{k_{i}}$ rings in both senses. It does not extend to direct sums of $p$-rings, where $p$ takes on at least two distinct values, or to $J$-rings as illustrated by the following example.

Let $R=G F(2) \oplus G F(2) \oplus G F(3)$ and $S=G F(3) \oplus G F(3) \oplus G F(2)$. Each of these rings has the property that $x^{6}=x$ for each $x$ in the ring and End $R \simeq$ End $S$, but $R$ is not isomorphic to $S$.

\section{References}

[1] A. L. Foster, ' $p^{k}$-rings and ring-logics', Ann. Scu. Norm. Pisa 5 (1951) 279-300.

[2] A. L. Foster, 'Generalized 'Boolean' theory of universal algebras, Part I', Math. Z. 58 (1953) 306-336.

[3] N. Jacobson, Structure of Rings. (Amer. Math. Soc. Colloquium, Vol. 36, revised edition (1964)).

[4] K. D. Magill, Jr., 'The Semigroup of endomorphisms of a Boolean ring', J. Austral. Math. Soc. 11 (1970) 411-416.

[5] C. J. Maxson, 'On semigroups of Boolean ring endomorphisms', Semigroup Forum 4, (1972) 78-82.

[6] N. H. McCoy, 'Subrings of Direct Sums', Amer. J. Math, 60 (1938) 374-382.

[7] N. H. McCoy, Rings and Ideals. (Carus Math. Monographs (MAA) Vol. 8 (1948)).

USAF Academy, Colorado 80840

U. S. A.

and

North Carolina State University at Raleigh 27607

U. S. A. 